\title{
Ground-based detections of sodium in HD 209458b's atmosphere in two data sets
}

\author{
S. Albrecht and I. Snellen and E. de Mooij and R. Le Poole \\ Leiden Observatory, University of Leiden, \\ P.O. Box 9513, NL-2300 RA Leiden, The Netherlands \\ email: albrecht@strw.leidenuniv.nl
}

\begin{abstract}
We present two separate ground-based detections of sodium in the transmission spectrum of HD 209458b. First we reanalyzed an archival data set from the HDS spectrograph on Subaru, which shows sodium at a $>5 \sigma$ level. Secondly, our preliminary results of a UVES/VLT data set indicate sodium absorption at a similar level, although the data cover the eclipse only partially. Both results are fully consistent with the HST results of Charbonneau et al. (2002). The Na D absorption feature seems to be resolved in the narrowest passband.
\end{abstract}

\section{Introduction}

Planetary atmospheres are expected to absorb in a wavelength-dependent manner. A stellar spectrum observed through the atmosphere of a transiting planet carries the spectral imprint of the atomic and molecular species in that atmosphere. Comparing in-transit with out-of-transit spectra of the host star can therefore reveal information about the composition and structure of the planet's atmosphere (e.g. Seager \& Sasselov 2000). For hot jupiters two prominent absorption features are expected in the visible wavelength band (Brown 2001); sodium absorption around the NaD lines (5889 $\AA$ and $5895 \AA$ ), and absorption by potassium $(7665 \AA$ and $7699 \AA)$. Charbonneau et al. (2002) were the first to detect the sodium feature in the transmission spectrum of an extra solar planet, HD 209458b. Recently, Redfield et al. (2008) detected sodium absorption by the atmosphere of HD 189733b via transmission spectroscopy from the ground.

In this paper we present two independent data sets which provide the first ground-based detection of sodium in the transmission spectrum of HD 209458b. First we will summarize the reanalysis and results of an archival data set from HDS/Subaru. The results have been recently published by Snellen et al. (2008). Subsequently, we will present our preliminary analysis and results of our UVES/VLT data set.

\section{HDS Subaru results}

HD 209458 was observed on the night of October 24, 2002, using HDS on the Subaru telescope. The observations have been described in Winn et al. (2004) and Narita et al. (2005). We concentrated on the 30 spectra from the red CCD $(5500 \AA<\lambda<6800 \AA)$, which were made with an exposure time of 500 seconds, a resolution of $R \approx 45000$, a SN ratio between 300 - 400 in the continuum, and of which nine where obtained before ingress and three after egress $\dagger$. After initial reduction and correction for the change in the blaze function we selected 59 strong stellar lines and the $\mathrm{NaD}$ doublet to perform our analysis. First, for each line, the line center was determined through Gaussian fitting.

$\dagger$ For a more in depth description of the data set see Winn et al. (2004), Narita et al. (2005), and Snellen et al. (2008). 

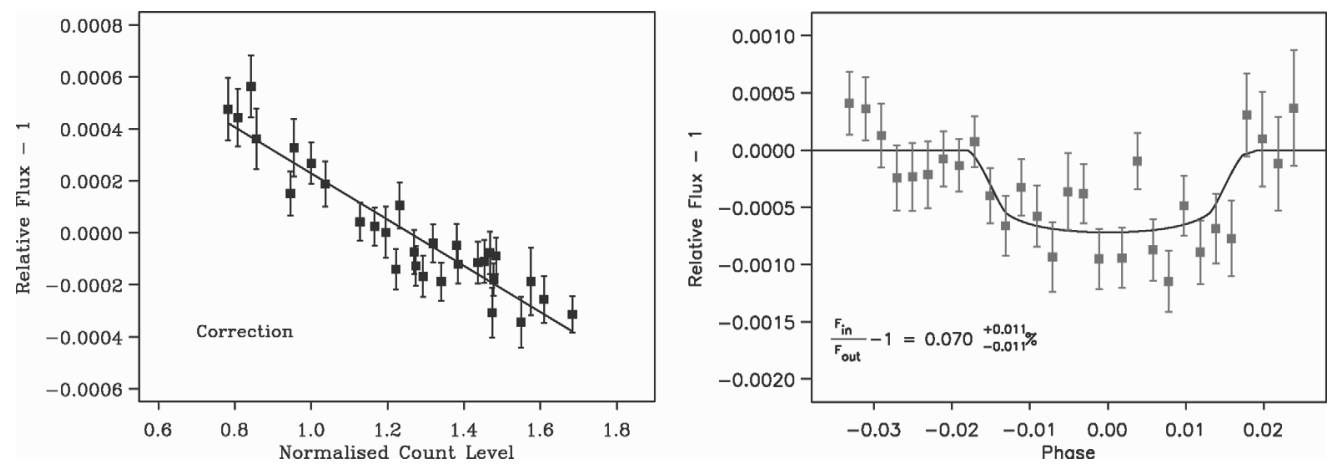

Figure 1. The left figure shows the weighted mean strength of 59 stellar lines over count level for the HDS - Subaru data. The right panel shows the transit photometry averaged over two passbands centered on the NaD lines each with a width of $1.5 \AA$. Reproduction of Fig. 4 (upper panel) and Fig. 3 (middle panel) in the paper by Snellen et al. (2008).

Subsequently, the total flux was integrated within a spectral band, and divided by the average of two equally wide bands to the left and right of the line. We found a strong correlation between the mean line depth of the 59 stronger stellar lines and the count level for each exposure (see left panel Fig. 1). This effect was attributed to a small nonlinearity in the conversion factor of the CCD and the electronics. If one assumes the conversion factor decreases slowly towards a high count a level, then high count levels are overestimated and low count levels are underestimated. If the count level for each exposure changes, one expects a behaviour as seen in the left panel of Fig. 1.

After correction for this effect and the influence of telluric lines (see Snellen et al. 2008) we derived the depth of the sodium signal due to the atmosphere of HD 209458b with $0.056 \pm 0.007 \%, 0.070 \pm 0.011 \%$, and $0.135 \pm 0.017 \%$ in the $2 \times 3.0 \AA, 2 \times 1.50 \AA$, and $2 \times 0.75 \AA$ passbands, respectively.

\section{UVES VLT observations}

We confirm the Subaru results with a new data set from UVES/VLT. In the night 13/14 August 2006 we obtained fifty-six high resolution spectra of HD 209458 using UVES on the UT2 telescope at ESO's Paranal observatory. Sixteen observations were taken during and forty after the transit $\dagger$. The spectra have a resolution of $\mathrm{R} \approx 55000$ and cover the spectral region from $3800 \AA<\lambda<9000 \AA$. After initial data reduction, using the ESOREX software package, we extracted spectra with a $\mathrm{S} / \mathrm{N}$ ratio between $200-300$ in the continuum.

As for the Subaru data the absorption due to sodium in the planet's atmosphere is determined by integrating the flux in two central bands centered on the $\mathrm{Na} \mathrm{D}$ lines and dividing by the flux in sidebands of the same width, adjacent on the left and right, respectively. Before this can be done, all other effects which change with time and that can influence the ratio between the central and sidebands, have to be excluded.

The blaze function of the spectrograph itself also changes over the course of a night. A good way to treat this effect is explained in Winn et al. 2004 and here we followed their approach by using the orders adjacent to the order of interest to correct for the change of the blaze function with time. Another instrumental effect for which we corrected is a non-linearity in the conversion factor of the CCD and its electronics, as discussed above,

$\dagger$ Observations $17-20$ are excluded from the analysis as they have been influenced by cloud cover. 

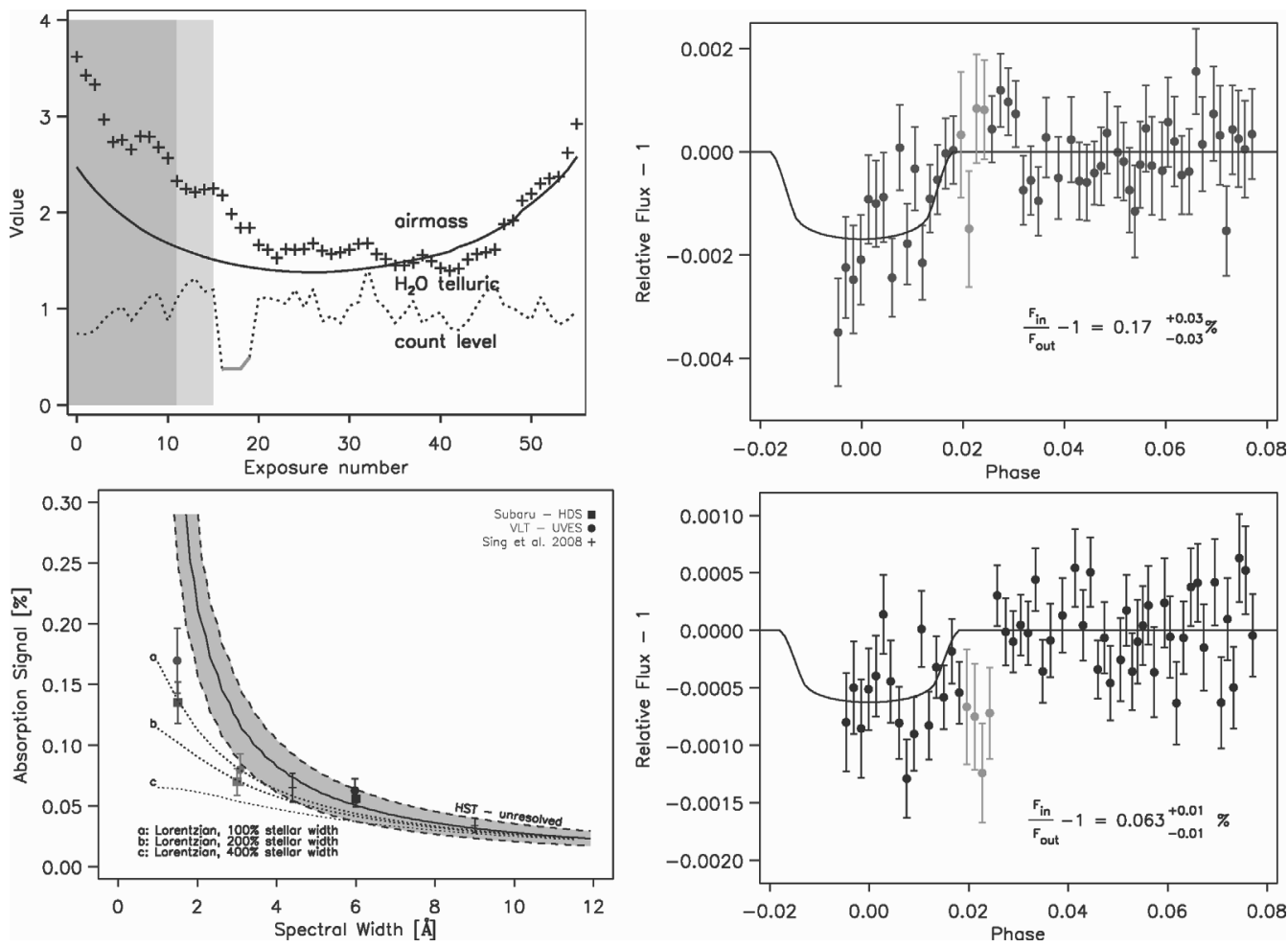

Figure 2. The upper left panel shows the environmental conditions during the observations of HD 209458 at the VLT in the night 13/14 August 2006. The grey areas indicate the timing of the planetary transit and period of egress. The top and bottom right panels show the transit photometry averaged over two passbands centered on the Na D lines each with a width of $0.74 \AA$ and $3.0 \AA$, respectively. Our results are presented in the bottom left panel: The six measurements of the Na D absorption in three different passband of the Subaru and UVES data are displayed as filled squares and circles, respectively. See text for further explanation.

and which is also present in the VLT data set. The dotted line in the upper left panel of Fig. 2 shows the change in the normalized counts per exposure. This effect is $\sim 3$ times smaller in the VLT data set (lower red CCD) than in the Subaru data set.

For ground-based observations, changes in telluric features, such as $\mathrm{H}_{2} \mathrm{O}$ lines around the $\mathrm{NaD}$ doublet and sodium absorption by the atmospheric sodium layer, can be problematic. Note that the regions in the earth atmosphere where the telluric water lines and the telluric sodium absorption originate are not identical and therefore the change of these telluric features over time can differ. In the Subaru data set, however, which was taken under good weather conditions, the strength of both features only depends on the airmass (see Fig. 2 in Snellen et al. 2008). One parameter was fitted which describes the influence of both telluric features on the stellar spectra in the chosen passbands. The weather conditions during the observations of HD 209458 at the VLT have been less stable (upper left panel Fig. 2). The telluric water lines (crosses) do not follow the airmass (solid line). Therefore two variables have to be determined which describe the influence of 1) the water lines and 2) the telluric sodium layer on the spectra in the passbands. This is done via linear fits to the residuals of the transmission spectroscopy for observations out of transit. The first coefficient scales proportionally to the strong telluric water lines shown in Fig. 2. The second scales, as for the Subaru data set, proportional to the 
airmass. The coefficients which minimize the scatter in the transmission spectroscopy of the $\mathrm{NaD}$ lines out of transit are then applied to all observations.

\section{UVES VLT Results}

For the new VLT observations the mean relative depths around the two NaD lines due to the planetary atmosphere are measured to be $0.063 \pm 0.01 \%, 0.08 \pm 0.013 \%$, and $0.17 \pm 0.027 \%$ in the $2 \times 3.0 \AA, 2 \times 1.50 \AA$, and $2 \times 0.75 \AA$ passbands, respectively (see right panels of Fig. 2). These depths are, within their uncertainties, consistent with our Subaru values, the results by Charbonneau et al. (2002), and the HST data reanalyzed by Sing et al. (2008). Fig. 2 lower left panel shows our results, filled squares and circles, the results by Sing et al. (2008), crosses, and as solid line and grey band, the HST measurement by Charbonneau et al. (2002) assuming it is completely unresolved and within our passbands. It seems the absorption of planetary sodium is partly resolved out in the narrowest band passes. Therefore, we have plotted three dotted lines, $a, b, c$, which indicate the $\mathrm{NaD}$ absorption levels as a function of passband expected from the HST measurements, assuming that the atmospheric absorption feature is Lorentzian shaped with a width equal to, $2 \times$ and $4 \times$ the stellar width of the $\mathrm{Na} \mathrm{D}$ doublet.

A crucial part of our data analysis is the removal of the influence of the telluric $\mathrm{H}_{2} \mathrm{O}$ lines which do not directly scale with the airmass - in contrast to that found in the Subaru data. As a consistency check we used the telluric line list of Lundstrom et al. 1991 to construct a synthetic telluric spectrum. For each observation we determined the strength of this spectrum by means of a $\chi^{2}$ fit to prominent telluric $\mathrm{H}_{2} \mathrm{O}$ features adjacent to the $\mathrm{Na} \mathrm{D}$ lines. The removal of this spectra leads to similar results as the former method. A further consistency check was performed to see if our correction for the non-linearity of the CCD is sensible. We correlated the depths of $\mathrm{NaD}$ lines with depths of other strong lines instead of removing the non-linearity from the spectra. Again this lead to similar results as before.

\section{Conclusions}

While the results of our UVES/VLT data are preliminary, we do detect absorption of sodium at a level consistent with Subaru and HST (Charbonneau et al. 2002) data. This indicates that with the correct reduction and analysis methods it becomes possible to study spectral transmission features from extra solar planets routinely from the ground.

\section{References}

Brown, T. M. 2001, ApJ, 553, 1006

Charbonneau, D., Brown, T. M., Noyes, R. W., \& Gilliland, R. L. 2002, ApJ, 568, 377

Lundstrom, I., Ardeberg, A., Maurice, E., \& Lindgren, H. 1991, A\&AS, 91, 199

Narita, N., Suto, Y., Winn, J. N., et al. 2005, PASJ, 57, 471

Redfield, S., Endl, M., Cochran, W. D., \& Koesterke, L. 2008, ApJL, 673, L87

Seager, S. \& Sasselov, D. D. 2000, ApJ, 537, 916

Snellen, I. A. G., Albrecht, S., de Mooij, E. J. W., \& Le Poole, R. S., arXiv:0805.0789v1

Sing, D. K., Vidal-Madjar, A., Desert, J. M., et al. arXiv0802.3864v2

Winn, J. N., Suto, Y., Turner, E. L., et al. 2004, PASJ, 56, 655 